\title{
Heart Rate Variability Associated with Rapid Eye Movements during Sleep
}

\author{
M Hoshiyama ${ }^{1,2}$, A Hoshiyama ${ }^{2}$ \\ ${ }^{1}$ The Univerisity of Tokyo, Tokyo, Japan \\ ${ }^{2}$ Meisei University, Tokyo, Japan
}

\begin{abstract}
To understand the association of heart rate control with rapid eye movement, we studied heart rate and eye movement continuously during sleep in 10 young, healthy subjects who do not suffer from any sleep disorder. The study took place over 3 nights in a sleep room. Polysomnographic recordings included electrooculogram (EOG), EEG, chin electromyogram (EMG) and ECG. The first and second nights were used for habituation, and the data obtained on the third night was used for analysis. Sleep stages were scored using standard $R-K$ criteria. Each eye movement was extracted from EOG with semi-automated algorithm followed by physician's confirmation. Population averaged results for DFA exponents of HRV were around 1/2 for non-REM sleep and 0.82 for REM sleep. Most notably, instantaneous heart rate showed distinctive increase within $30 \mathrm{sec}$ of the rapid eye movements $(p=0.04)$ with modification by the number of succeeding rapid eye movements.
\end{abstract}

\section{Introduction}

Normal sleep is characterized by cycles of non-rapideye-movement followed by rapid-eye-movement (REM) sleep, with typically 5-6 cycles of durations approximately 90 minutes over the course of the night. While heart rate during sleep has been studied in the past for both rapid eye movement (REM) sleep and non-REM sleep [1-4], there remains a lack of consensus whether the rapid eye movement during sleep elicit consistent heart rate response, partly due to the difficulty in extracting eye movements from noisy electrooculogram (EOG) signals. We characterized the association of heart rate control with rapid eye movement by studying heart rate and eye movement continuously during sleep in 10 young, healthy subjects.

\section{Methods}

We studied 10 young healthy subjects, who did not suffer from any sleep disorder. Subjects had regular sleep habits. None of the subjects were taking any medication.
The study took place over 3 nights in a sound-proof, airconditioned (22-24Celcius) sleep room. Polysomnographic recordings included EOG, electroencephalogram (EEG), chin electromyogram (EMG) and ECG. Subjects went to bed at their regular bedtime and got up spontaneously. The first and second nights were used for habituation, and the data obtained on the third night was used for analysis.

The HR was obtained from ECG recordings during sleep. Sleep stages were scored using standard Rechtschaffen Kales criteria [5]. Eye electrodes are attached on the upper-outer edge of the right eye and lower-outer edge of the left eye, with a common electrode on the left mastoid. If both eyes move in the same direction, the polarity of the both eye potentials will be opposite, and vise versa. In order to avoid detecting transmitted EEG potentials from frontal lobes, two signals that have the same polarity are omitted. EOG data were digitized with a sampling rate of $64 \mathrm{~Hz}$. Each eye movement was extracted from EOG recordings using semi-automated algorithm followed by physician's confirmation (Fig. 1).

We computed the detrended fluctuations analysis (DFA) exponents [6-9] of HRV during non-REM sleep and REM sleep. Exponents are calculated from linear fits to $\log$ - $\log$ plots of $\mathrm{F}(n)$ versus $n$ in the regime $70<\mathrm{n}<$ 300 . We chose this fitting range to be above the regime of short-range correlations related to breathing and below the $n$ values where statistical errors become too large due to the finite length of the sleep stages.

We analyzed instantaneous heart rate before and after the onset of the rapid eye movements. The maximum HR of moving 10 second window average between the onset and 30 second after the onset of rapid eye movements are compared with the 30 seconds average HR before the onset. 


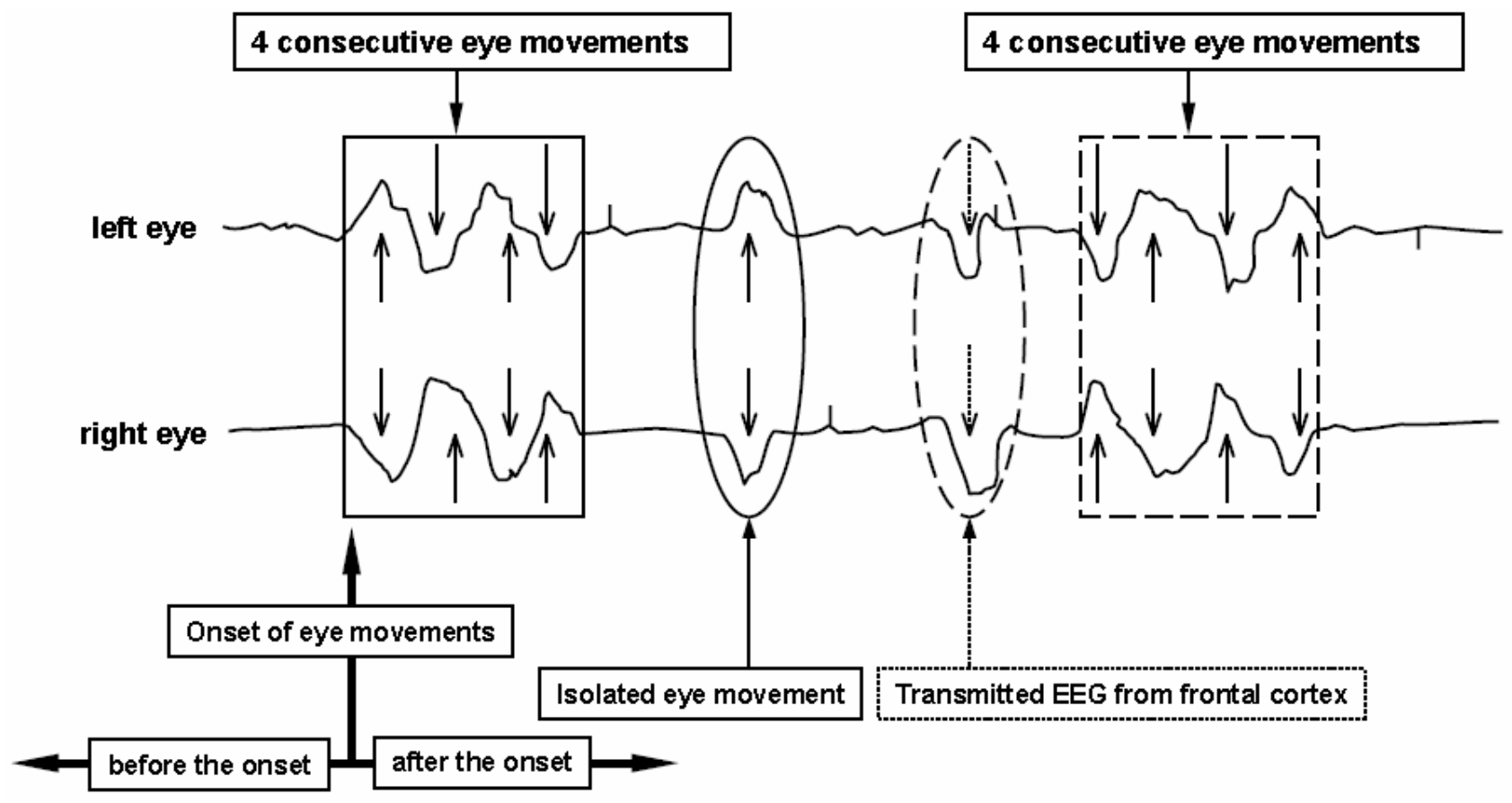

Figure 1. Eye electrodes are attached on the upper-outer edge of the right eye and lower-outer edge of the left eye, with a common electrode on the left mastoid. If both eyes move in the same direction, the polarity of the both eye potentials will be opposite, and vise versa. In order to avoid detecting transmitted EEG potentials from frontal lobes, two signals that have the same polarity are omitted. We compared heart rate before and after rapid eye movements.

\section{Results}

Population averaged results for heart rate were not significantly different between light and deep sleep, where $(56.0,2.2)$ for light sleep, $(55.8,1.8)$ for deep sleep and $(58.5,2.0)$ for REM sleep. (mean, SEM) [/min] (Fig. 2).

Population averaged results for DFA exponents of HRV were not significantly different between light and deep sleep. The histograms are centered around 0.5 for non-REM sleep, showing almost no correlations. DFA exponents of HRV were around 0.82 for REM sleep, implicating long-range correlations (Fig. 3).

The correlations during REM sleep were similar to those during awake, which were consistent with previous studies [10].

Instantaneous heart rate showed distinctive increase within $30 \mathrm{sec}$ of the rapid eye movements $(\mathrm{p}=0.04)$ with modification by the number of succeeding rapid eye movements (Fig. 4).

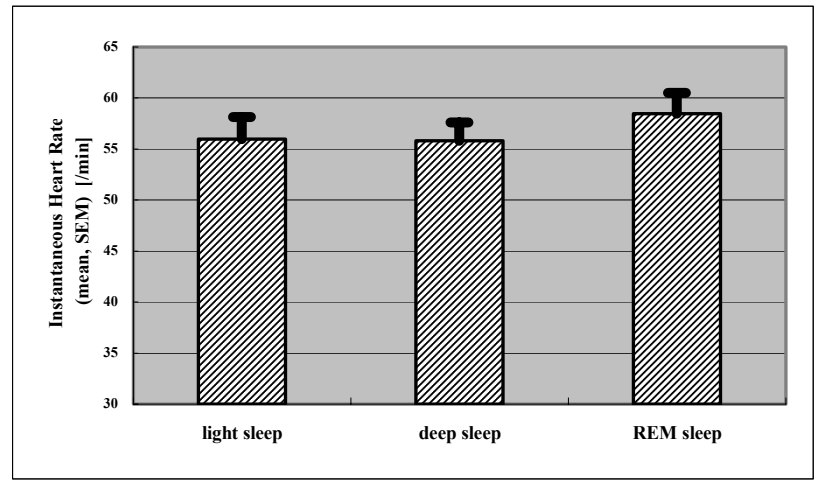

Figure 2. Population averaged results for heart rate were not significantly different between light and deep sleep. Standard errors of the mean are presented as error bars. 


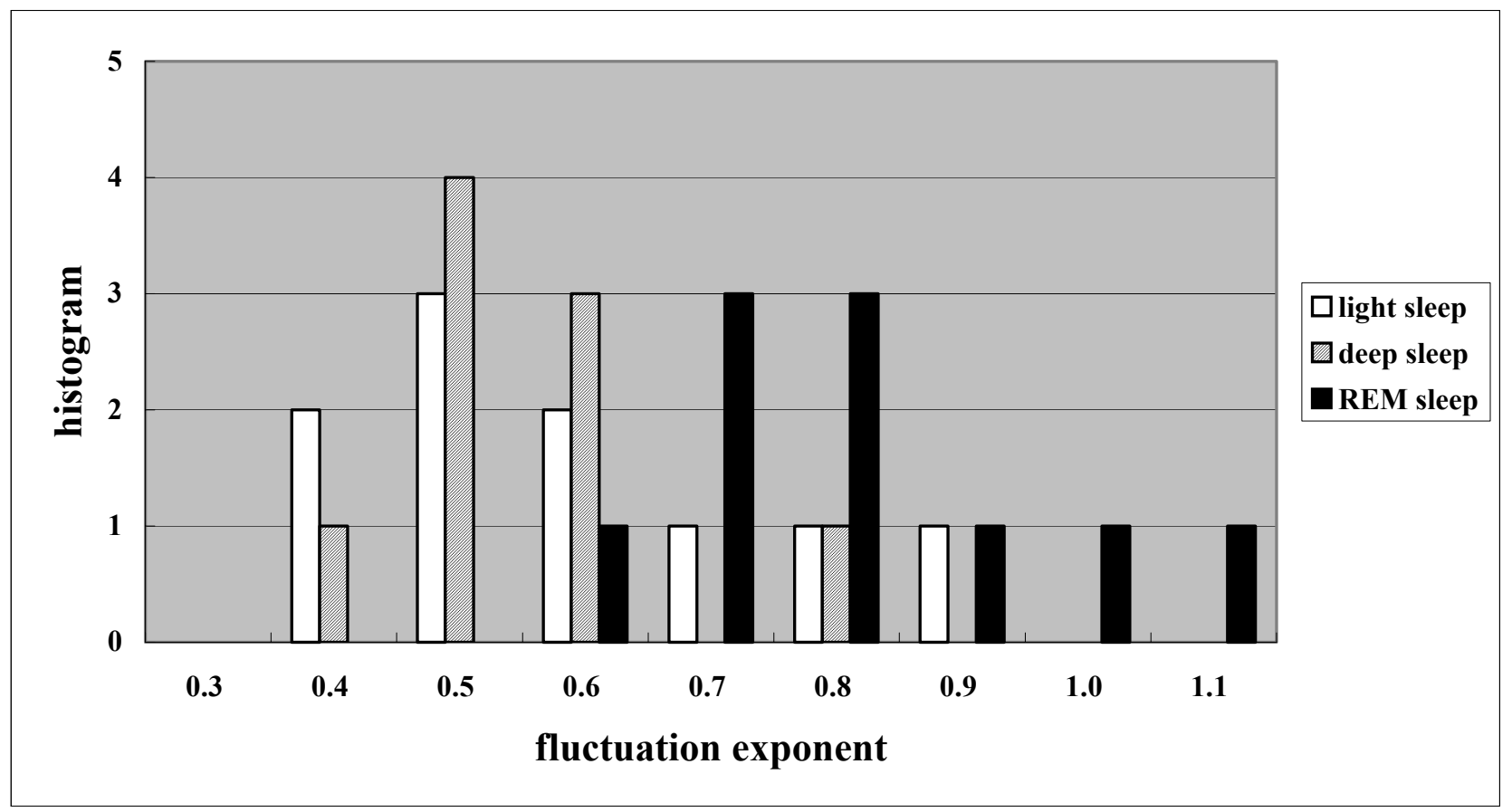

Figure 3. Histograms of DFA exponents of HRV for light sleep, deep sleep and REM sleep. DFA exponents were around 0.5 for non-REM (light and deep) sleep and 0.82 for REM sleep.

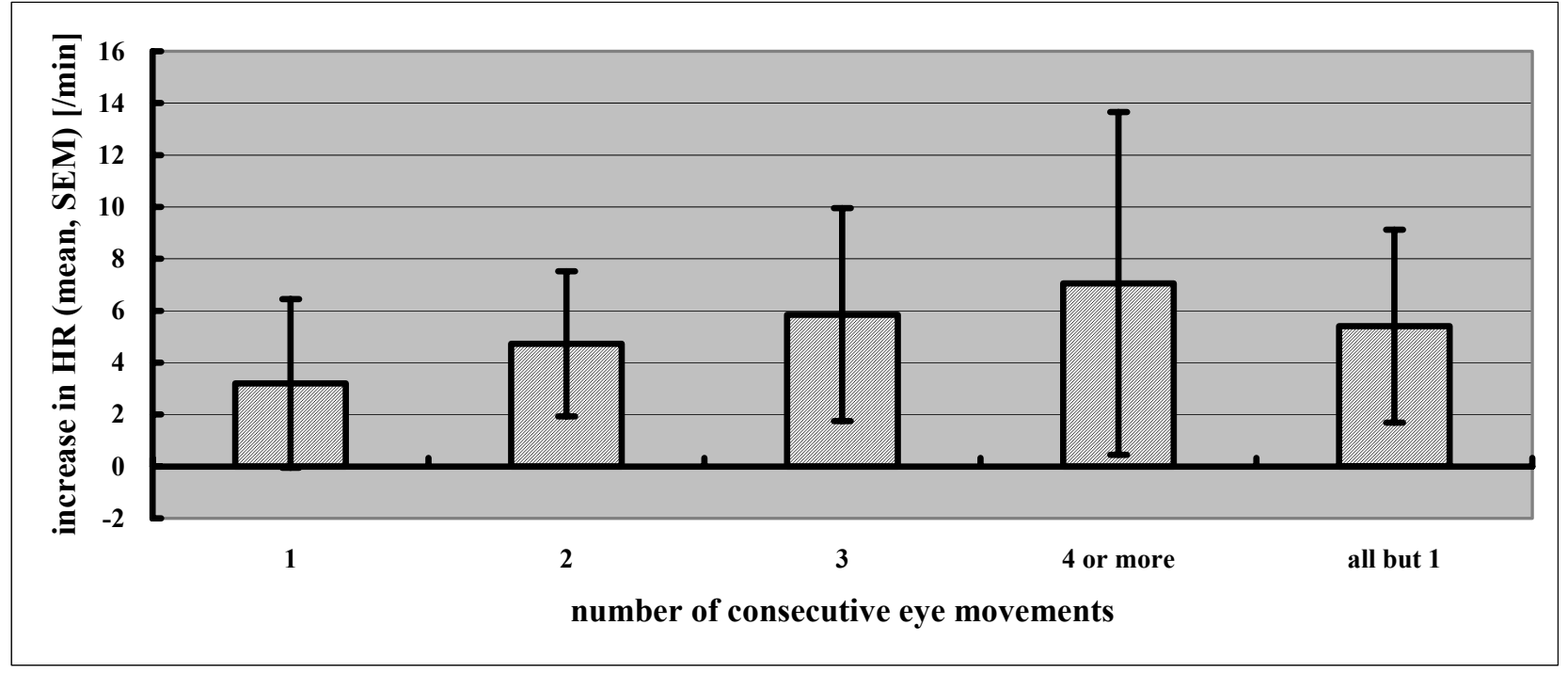

Figure 4. Histograms of increase in heart rate after eye movement. Instantaneous heart rate showed distinctive increase within $30 \mathrm{sec}$ of the rapid eye movements $(\mathrm{p}=0.04)$ with modification by the number of succeeding rapid eye movements. Standard errors of the mean are presented as error bars.

\section{Discussion and conclusions}

During sleep, eye movements of REM sleep are accompanied by various primary brainstem discharges. Cortical activation is invoked by high levels of regular neuronal discharge in the midbrain through thalamus into 
cortex, in which activation, Steriade has shown an important role of the cholinergic neurons [11]. The visual occipital cortex is stimulated by Pontine-GeniculateOccipital (PGO) waves that originate in the pons, with information including both the timing and the direction of eye movement $[12,13]$. Reticular cells in the medulla fire at a high regular rate, inhibiting the spinal motor neurons which leads to motor paralysis. Pontine neurons fire in bursts, inhibiting sensory nerve terminals in the spinal cord which leads to diminished sensation. Associated with these orchestrated neuronal systems of cortical activation, PGO waves, motor paralysis and diminished sensation, pontine reticular cells discharge in bursts, which directly excite nearby oculomotor neurons, resulting in ocular saccades [12].

We attribute the increase in heart rate after rapid eye movement to the autonomic arousal mechanism which is associated with this orchestrated activation of neuronal systems around pontine systems.

\section{Acknowledgements}

We are grateful to J. A. Hobson for helpful suggestions on sleep physiology.

\section{References}

[1] Baharav A, Kotagal S, Gibbons V, Rubin BK, Pratt G, Karin J, Akselrod S. Fluctuations in autonomic nervous activity during sleep displayed by power spectrum analysis of heart rate variability. Neurology 1995;45:1183-7.

[2] Bonnet MH, Arand DL. Heart rate variability: sleep stage, time of night, and arousal influences. Electroencephalogr 1997;102:390-6.

[3] Otzenberger H, Cronfier C, Simon C, Charloux A, Ehrhart J, Piquard F, Brandenberger G. Dynamic heart rate variability. Am J Physiol Heart Circ Physiol 1998;275:H946-50.
[4] Vaughn BV, Quint SR, Messenheimer JA, Robertson KR. Heart period variability in sleep. Electroencephalogr Clin Neurophysiol 1995;94:155-62.

[5] Rechtschaffen A, Kales A. A Manual of Standardized Terminology, Techniques and Scoring System for Sleep Stages of Human Subjects. Los Angels: Brain information Service/Brain Ressearch Institute, 1968.

[6] Peng CK, et al. Quantification of scaling exponents and crossover phenomena in nonstationary heartbeat time series. Chaos 1995;5:82-87.

[7] Bak P, Tang C, Wiesenfeld K. Self-organized criticality. Phys Rev Lett 1987;59:381-384.

[8] Stanley HE. Introduction to Phase Transitions and Critical Phenomena. London:Oxford University Press,1971.

[9] Goldberger AL. Non-linear dynamics for clinicians: chaos theory, fractals, and complexity at the bedside. Lancet 1996;347:1312-14.

[10] Bunde A, Havlin S, Kantelhardt JW, Penzel T, Peter JH, Voigt K. Correlated and Uncorrelated Regions in HeartRate Fluctuations during Sleep. Phys Rev Lett 2000;85:3736-9.

[11] Dossi RC, Pare D, Steriade M. Short-lasting nicotinic and long-lasting muscarinic depolarizing responses of thalamocortical neurons to stimulation of mesopontine cholinergic nuclei. J Neurophysiology 1991;65:393-406.

[12] Nelson JP, McCarley RW, Hobson JA. REM sleep burst neurons, PGO waves, and eye movement information. J Neurophysiology 1983;50:784-97.

[13] Peigneux P, Laureys S, Maquet P. Generation of rapid eye movements during paradoxical sleep in humans. Neuroimage 2001;14:701-8.

Address for correspondence

Masaki Hoshiyama, M.D., Ph.D.

Omachi 1-9-12, Kamakura, Kanagawa, Japan

M.Hoshiyama@gmail.com 